\title{
One of the Rare Reasons of Abdominal Pain-Chilaiditi's Syndrome
}

\author{
Eylem Kuday Kaykısız', Özgür Özer², Zübeyir Dalgıç \\ ${ }^{1}$ Clinic of Emergency Medicine, Bitlis State Hospital, Bitlis, Turkey \\ ${ }^{2}$ Clinic of Radiology, Bitlis State Hospital, Bitlis, Turkey
}

\begin{abstract}
Chilaiditi's sign is the anatomical description of the interposition of the colon between the liver and the diaphragm; when it is accompanied with clinical symptoms, it is known as Chilaiditi's syndrome. Most commonly, it is an asymptomatic radiological finding and is considered a rare entity, and therefore, it is often misdiagnosed in clinical practice; however, it may be accompanied by a series of severe complications. Here we report a patient with Chilaiditi's syndrome, owing to its rarity, and the typical radiological findings of this syndrome. A 22-year-old female was admitted to the hospital, presenting with a 2-day history of nausea and worsening right upper quadrant pain. Vital signs were stable. The physical examination revealed a soft abdomen with mild right upper abdominal tenderness. Laboratory assays showed no abnormalities. A plain abdominal X-ray showed an abnormal gas shadow in the subhepatic space and a segment of gaseous distended colon. A computarized tomography (CT) scan of the abdomen showed a loop of colon interpositioned between the liver and the right hemidiaphragm, mimicking free air. The patient consulted with a general surgery physician, and was admitted to the general surgery service to follow up on treatment and operation, if indicated. The patient was managed conservatively. During the course of her hospital stay, her abdominal pain resolved without surgical intervention. She was then able to tolerate a regular diet, and was discharged after two days of hospital stay. Although Chilaiditi's syndrome is not common, it is important, and can be easily mistaken for pneumoperitoneum. Most of the cases with Chilaiditi's syndrome can be resolved with nasogastric decompression and repeated laxatives. Surgical intervention is reserved for patients with signs of systemic toxicity or peritonitis. Owing to the rarity of this syndrome and its typical radiological findings, we aimed to present this case.
\end{abstract}

Keywords: Chilaiditi's syndrome, abdominal pain, colon interposition

\section{Introduction}

Chilaiditi's sign is the anatomical description of the interposition of the colon between the liver and the diaphragm. It was first described by Demetrius Chilaiditi in 1910 (1). Chilaiditi's sign has an incidence of $0.025 \%-0.28 \%$ worldwide, with a marked male predominance (male to female, $4: 1)(2,3)$. Most commonly, it is an asymptomatic radiological finding and is considered as a rare entity, and therefore, it is often misdiagnosed in clinical practice; however, it may be accompanied by a series of severe complications, such as bowel obstruction and perforation (4). The differential diagnosis of Chilaiditi's syndrome includes pneumoperitoneum, pneumobilia, and hepatic-portal venous gas. The therapy for Chilaiditi's syndrome consists of conservative nasogastric decompression and bed rest. Surgical intervention is rarely indicated (4).

\section{Case Presentation}

A 22-year-old female was admitted to the Emergency Department of the Bitlis State Hospital in December 2016, presenting with a 2-day history of nausea and worsening epigastric and right upper quadrant pain. The pain was sharp and radiated to the right shoulder. She denied dysphagia, early satiety, fever, chills, night sweats, melena, hematochezia, or any changes in her bowel habits. She did not recall having a similar experience before. The patient had no history of surgery. On original presentation, she was afebrile, with a blood pressure of $126 / 91 \mathrm{mmHg}$, pulse of 77 beats/min, respiratory rate of 20 beats/min, and oxygen saturation of $99 \%$ on room air. The physical examination revealed a soft abdomen with mild right upper abdominal tenderness; no rebound tenderness or muscle guarding was identified. The cardiovascular and respiratory exams were unremark- 


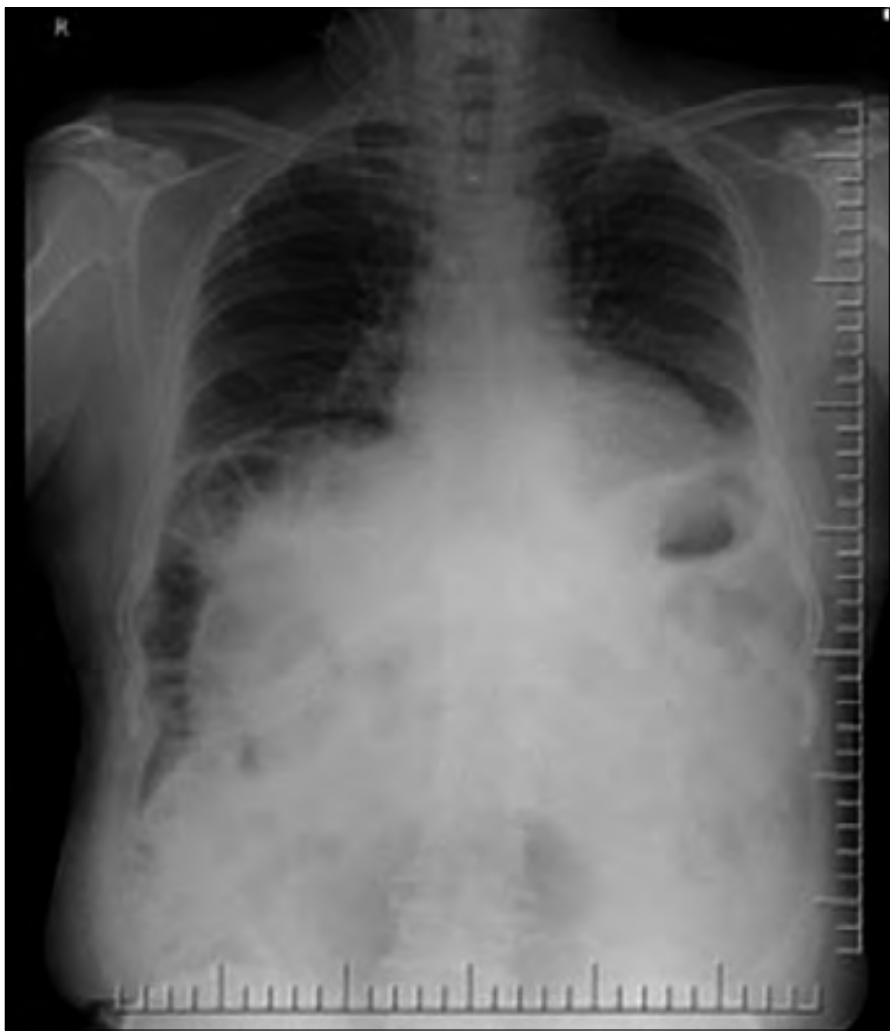

Figure 1. X-ray showed an abnormal gas shadow in the right subhepatic space, and a segment of gaseous distended colon, which was located on the right side of the abdominal cavity; this was interposed between the liver and the right diaphragm

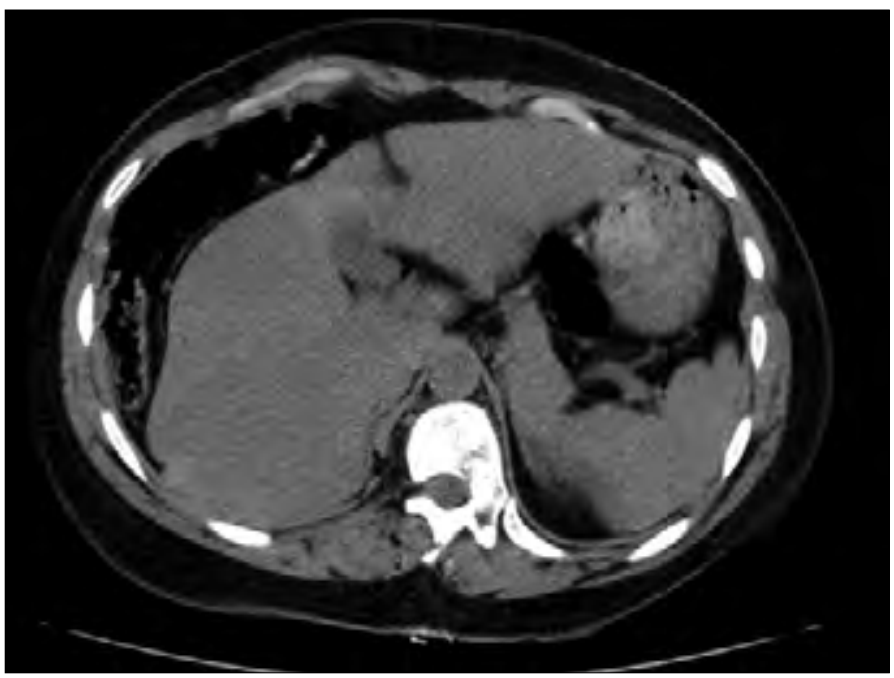

Figure 2. Computarized tomography (CT) scan of the abdomen and pelvis showed a loop of colon interpositioned between the liver and the right hemidiaphragm, mimicking free air

able. Laboratory assays, including hemogram, biochemistry, and full urinalysis showed no abnormalities.

A plain abdominal X-ray showed an abnormal gas shadow in the right subhepatic space, and a segment of gaseous distended colon, which was located on the right side of the abdominal cavity; this was interposed between the liver and the right diaphragm (Figure 1). Further imaging by a Computarized Tomography (CT) scan of the abdo- men and pelvis showed a loop of colon interpositioned between the liver and the right hemidiaphragm, mimicking free air (Figure 2). After that, the patient consulted with a general surgery physician, and was admitted to the general surgery service to follow up on treatment and operation, if indicated. The patient was managed conservatively with Intravenous (IV) fluid hydration and pain management. During the course of her hospital stay, her abdominal pain resolved without surgical intervention. She was then able to tolerate a regular diet, and was discharged after two days of hospital stay.

Informed consent was obtained from the patient.

\section{Discussion}

Chilaiditi's sign is the anatomical description of the interposition of the colon between the liver and the diaphragm. When it is accompanied with clinical symptoms, such as abdominal pain, nausea, vomiting, and constipation, it is known as Chilaiditi's syndrome (4). Several causes, including absence of suspensory ligaments of the transverse colon, atrophic or small liver, segmental agenesis of the right lobe of the liver, abnormality of the falciform redundant mesocolon, redundant or dilated colon, and volvulus of the colon have been reported to be associated with Chilaiditi's syndrome $(5,6)$.

Chilaiditi's sign is often an incidental finding and is asymptomatic. Therefore, obtaining the medical history and performing a careful physical examination are important for surgeons. However, old patients or patients with dementia can scarcely state their complaints on their own, and it is also difficult to obtain their medical history or perform a thorough physical examination. In our case, the patient had 2 days of nausea and worsening epigastric and right upper quadrant pain, and was believed to have biliary colic. She was saved from a surgical procedure due to a careful examination.

Radiological studies may be helpful in this situation. Most of the patients with Chilaiditi's sign can be easily diagnosed via chest X-ray. However, the haustral folds of bowel loops may not be seen in a chest X-ray. Sato et al. (7) reported that ultrasound is helpful in diagnosing Chilaiditi's syndrome. Most of the cases that can be diagnosed by $X$-ray and CT of the abdomen provide more information. However, ultrasound may be helpful in distinguishing between Chilaiditi's syndrome and pneumoperitoneum. In our case, X-ray and ultrasound diagnostic tools were unremarkable, so we performed a CT scan. A CT scan of the abdomen and pelvis, with intravenous contrast, showed a loop of colon interpositioned between the liver and the right hemidiaphragm, mimicking free air.

The management of Chilaiditi's syndrome varies due to its different etiologies, and includes both, operative and nonoperative approaches. Saber and Boros (5) reported that $26 \%$ of patients needed operative management, while the majority required nonoperative treatment, including bowel decompression and repeated radiography. If the symptoms were persistent, surgical intervention may be indicated. Bowel decompression may be both, diagnostic and therapeutic (5). In our case, the patient was managed conservatively with IV fluid hydration and pain management.

\section{Conclusion}

Although Chilaiditi's syndrome is not common, it is important, and can be easily mistaken for pneumoperitoneum. Most of the patients with Chilaiditi's sign are found incidentally. When it is accom- 
panied with clinical symptoms, it is known as Chilaiditi's syndrome. Most of the cases with Chilaiditi's syndrome can be resolved with nasogastric decompression, repeated laxatives, and enemas. Surgical intervention is reserved for patients with signs of systemic toxicity or peritonitis. In our case, the patient was initially believed to have biliary colic pain. However, considering the possibility of Chilaiditi's syndrome protected the patient from an unnecessary procedure, such as operation. Owing to the rarity of this syndrome and the typical radiological findings associated with it, we aimed to present this case.

Informed Consent: Written informed consent was obtained from patient who participated in this study.

Peer-review: Externally peer-reviewed.

Conflict of Interest: No conflict of interest was declared by the authors.

Financial Disclosure: The authors declared that this study has received no financial support.

\section{References}

1. D. Chilaiditi, "Zur Frage der Hepatoptose und Ptose im Allgemeinen im Anschlussan Drei Falle von Temporarer, Partieller Lebererlagerung," Fortschr Geb Rontgenstr Nu- klearmed Erganzungsband 1910; 16: 173-208.

2. Alva S, Shetty-Alva N, Longo WE. Image of the month. Chilaiditi sign or syndrome. Arch Surg 2008; 143: 93-4 . [CrossRef]

3. Yin AX, Park GH, Garnett GM, Balfour JF. Chilaiditi syndrome precipitated by colonoscopy: a case report and review of the literature. Hawaii J Med Public Health 2012; 71: 158-62.

4. Orangio GR, Fazio VW, Winkelman E, McGonagle BA. The Chilaiditi Syndrome and associated volvulus of the transverse colon. An indication for surgical therapy. Dis Colon Rectum 1986; 29: 653-6. [CrossRef]

5. Saber AA, Boros MJ. Chilaiditi's syndrome: what should every surgeon know? Am Surg 2005; 71: 261-3.

6. Kurt Y, Demirbas S, Bilgin G, Aydin Y, Akin L, Yildiz M, et al. Colonic volvulus associated with Chilaiditi's syndrome: report of a case. Surg Today 2004; 34: 613-5. [CrossRef]

7. Sato $M$, Ishida H, Konno $K$, Hamashima $Y$, Naganuma H, Komatsuda T, et al. Chilaiditi syndrome: sonographic findings. Abdom Imaging 2000; 25: 397-9. [CrossRef] 